Supporting Information for

\title{
Multilayered Microfluidic Paper-Based Devices: Characterization, Modeling, and Perspectives
}

Robert B. Channon, ${ }^{1 \dagger}$ Michael P. Nguyen, ${ }^{1 \dagger}$ Charles S. Henry, ${ }^{1,2,3 *}$ David S. Dandy ${ }^{2,3 *}$

${ }^{1}$ Department of Chemistry, ${ }^{2}$ Department of Chemical \& Biological Engineering, ${ }^{3}$ School of Biomedical Engineering, Colorado State University, CO, USA

${ }^{\dagger}$ These authors contributed to equally to this work

*Corresponding authors

\section{TABLE OF CONTENTS}

S1. Saturation of paper walls during flow in multilayered $\mu$ PADs

S2. Side view still images during flow in multilayered $\mu$ PADs with different paper thickness

S3. Effect of sampling method on velocity in multilayered $\mu$ PADs

S4. Evaporation of water from a multilayered $\mu \mathrm{PAD}$

S5. Model Development 
S1. Saturation of paper walls during flow

To assess the saturation of the paper walls during flow in multilayered devices, the velocity of liquid perpendicular to flow was calculated. This was carried out by measuring the time the liquid reaches the channel side and outside of the paper walls, for 7 set distances down the channel (splitting each image up into 7 quadrants normal to the direction of flow and measuring the velocity at each position). Note this assumes wetting of the paper perpendicular to the direction of flow, i.e., liquid wets from the gap into the paper and with a negligible contribution from liquid already within the paper behind the fluid front. Also note that some variability is observed in velocities down the channel, likely due variations in (i) capillary density, (ii) capillary orientation, and (iii) paper thickness as seen in Figure S3.
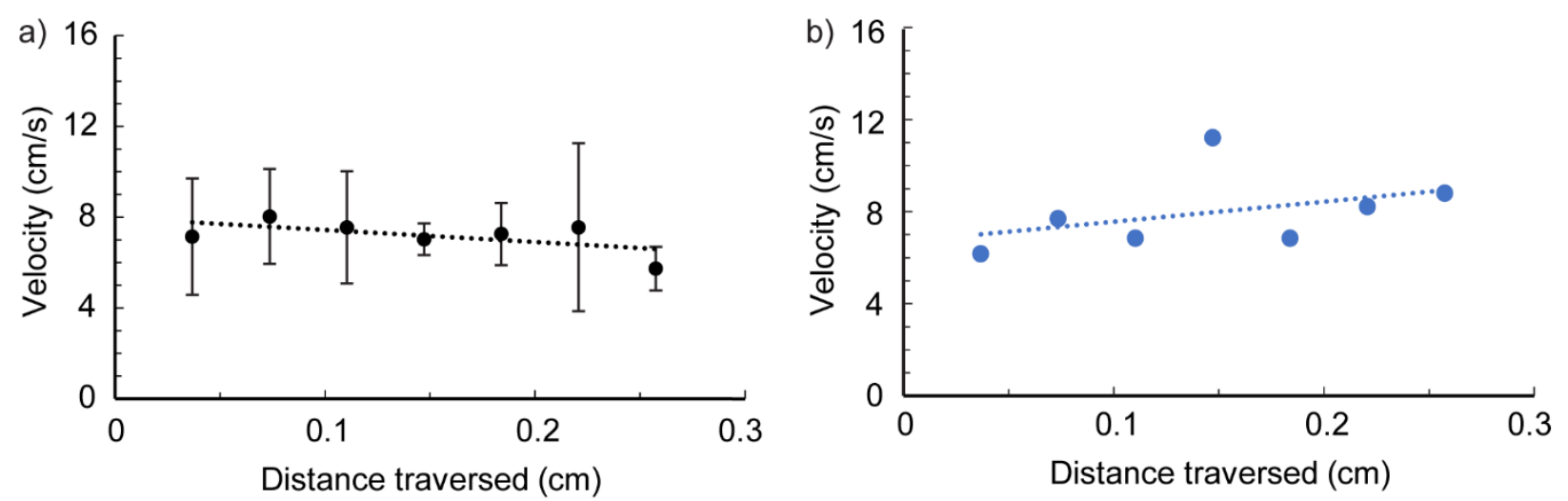

Figure S1. Velocity-distance plots of the fluid front wicking into a) Whatman 1 chromatography paper (n $=3,180 \mu \mathrm{m}$ paper thickness) and b) 3MM paper ( $n=1,340 \mu \mathrm{m}$ paper thickness) multilayered $\mu$ PADs, based on image analysis corresponding with Figure 2 in the main text (both 5DST / $390 \mu \mathrm{m}$ gap height).

The effect of paper thickness on flow characteristics can also be examined. For the same conditions listed in the Figure S1 caption, application of Equation (S20) or Equation (10) yields the following predictions for velocity over the same time span considered in Figure 6c.

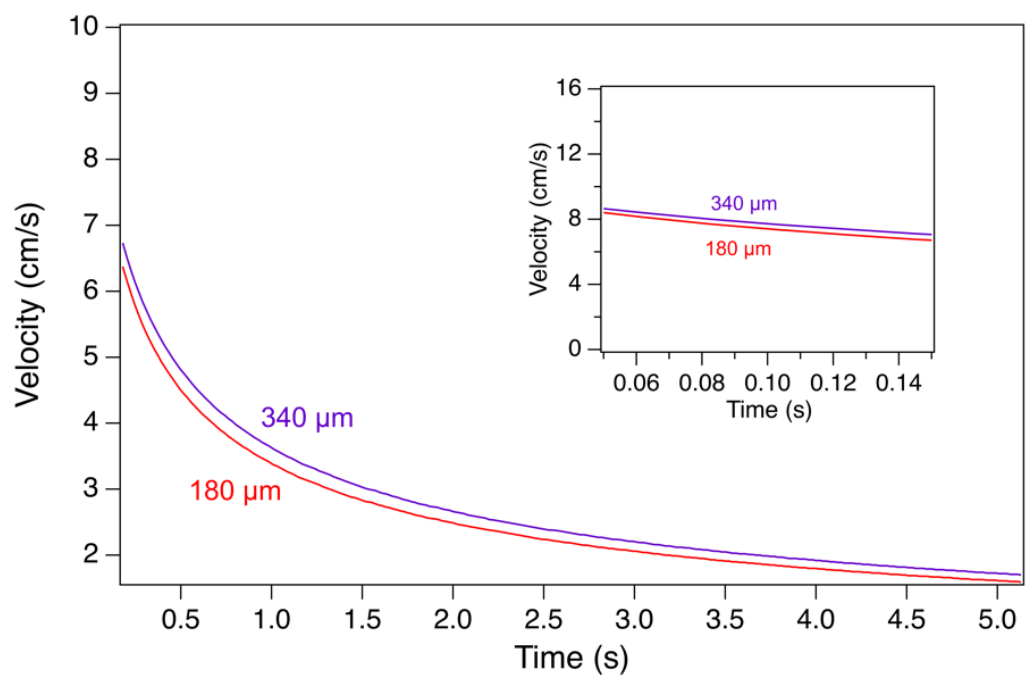

Figure S2. Predicted imbibition velocity in the device for Whatman 1 chromatography paper $\left(t_{h}=180 \mu \mathrm{m}\right)$ and $3 M M$ paper $\left(t_{h}=340 \mu \mathrm{m}\right)$ with a $390 \mu \mathrm{m}$ gap height. The inset shows the comparison at short penetration distances. 
For the first $\sim 0.10 \mathrm{~s}$ of penetration, that is, within $0.3 \mathrm{~cm}$ of the inlet, the front velocity in the device with $3 \mathrm{MM}$ paper is an average of $3 \%$ greater than with the Whatman 1 paper. The difference increases to $\sim 7 \%$ at longer times/penetration distances. And these velocities are higher than predicted and observed with the $96 \mu \mathrm{m}$-thick paper used throughout the rest of the study. The reason for the increase in local velocity with paper thickness is likely due to a decrease in the magnitude of the viscous dissipation effect and the mathematical solution's nonlinear dependence on paper thickness $t_{h}$.

S2. Frames captured from videos showing the fluid front in multilayered $\mu$ PADs with different paper thickness

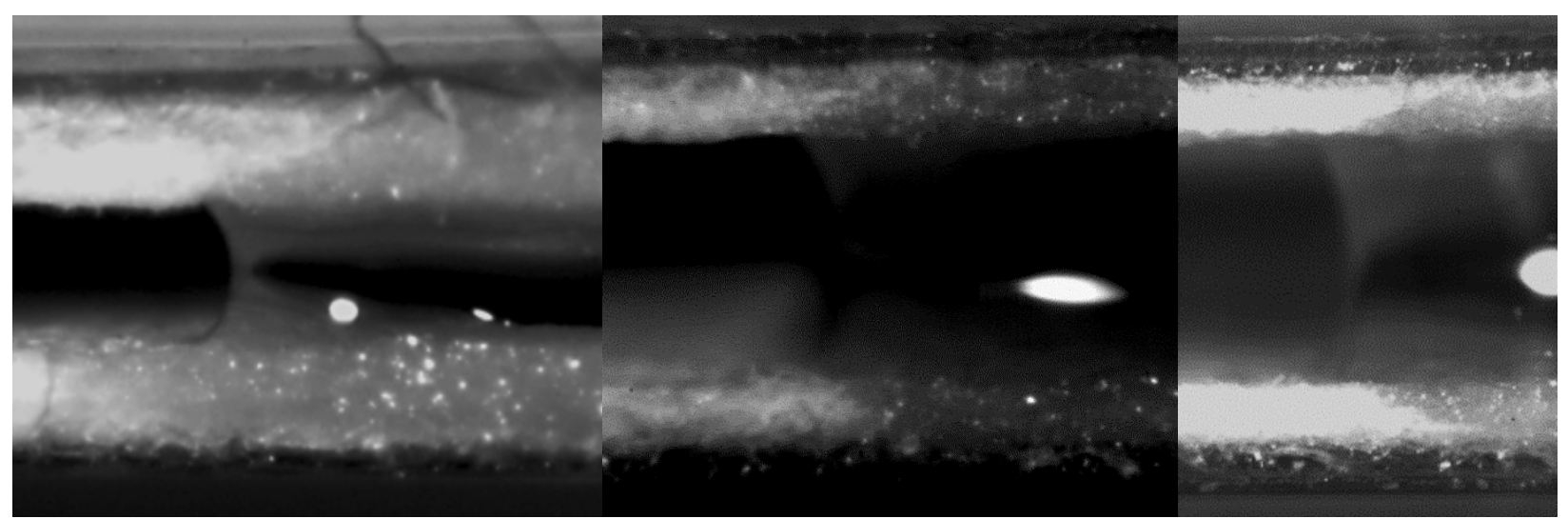

Figure S3. Still images of three different $\mu P A D s$ (390 $\mu \mathrm{m}$ gap heights), with water flowing from right to left across the image. Note that the bright circular spots to the right of the fluid front are reflections from the laboratory lights. A video of this experiment is provided as electronic supporting information ("side on flow"). 
S3. Effect of sampling method on velocity in multilayered $\mu$ PADs

Figure S4 shows separated plots from Figure 4 of the main text, to illustrate varying decay of velocity over the first $2 \mathrm{~cm}$ of liquid flow, based on the sampling methods.
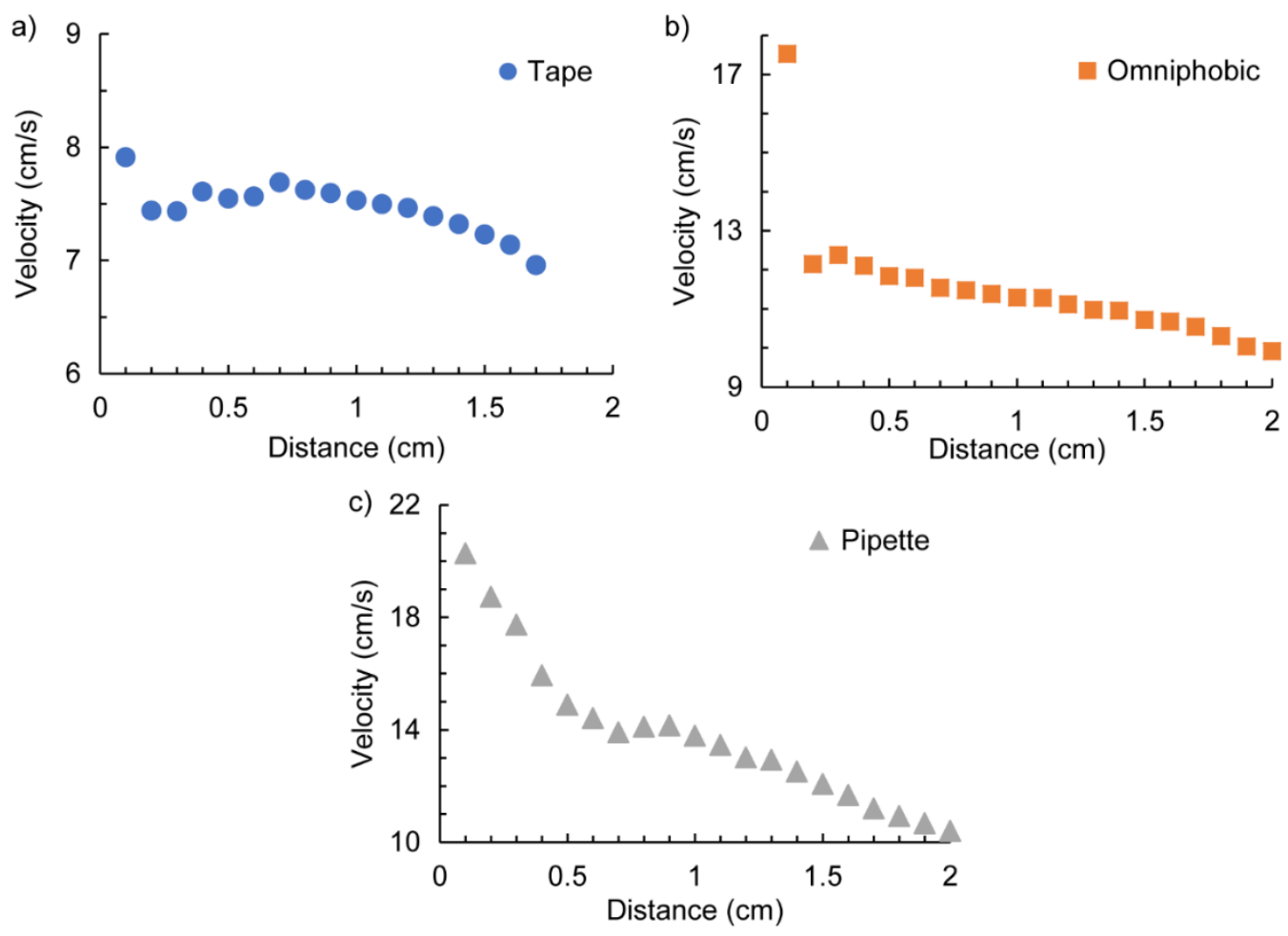

Figure S4. Velocity changes over distance transvered down multilayer $\mu$ PADs using different sampling methods a) addition through pipetting (grey triangles), b) connection to aliquots on a super omniphobic surface (orange squares), and c) connection to aliquots in tape wells (blue circles), $234 \mu \mathrm{m}$ gap height, Whatman $3 M M$ paper, $n=5$.

The following images (Figure S5) are representative snapshots of the high frame rate imaging carried out on multiple devices through the three sampling methods (tape, omniphobic, pipette). Note the longer times between liquid-paper contact and the solution initiating flow for the droplet approaches ( $a$ and $b$ ) compared to the pipette sampling due to the effect of droplet surface tension. Furthermore, note the ripples generated in Figure S4b when the droplet on omniphobic surface begins delivering liquid to the channel / gap. Videos of these sampling methods are provided online as electronic supporting information, namely "tape sampling", "pipette sampling", "omniphonic sampling". 

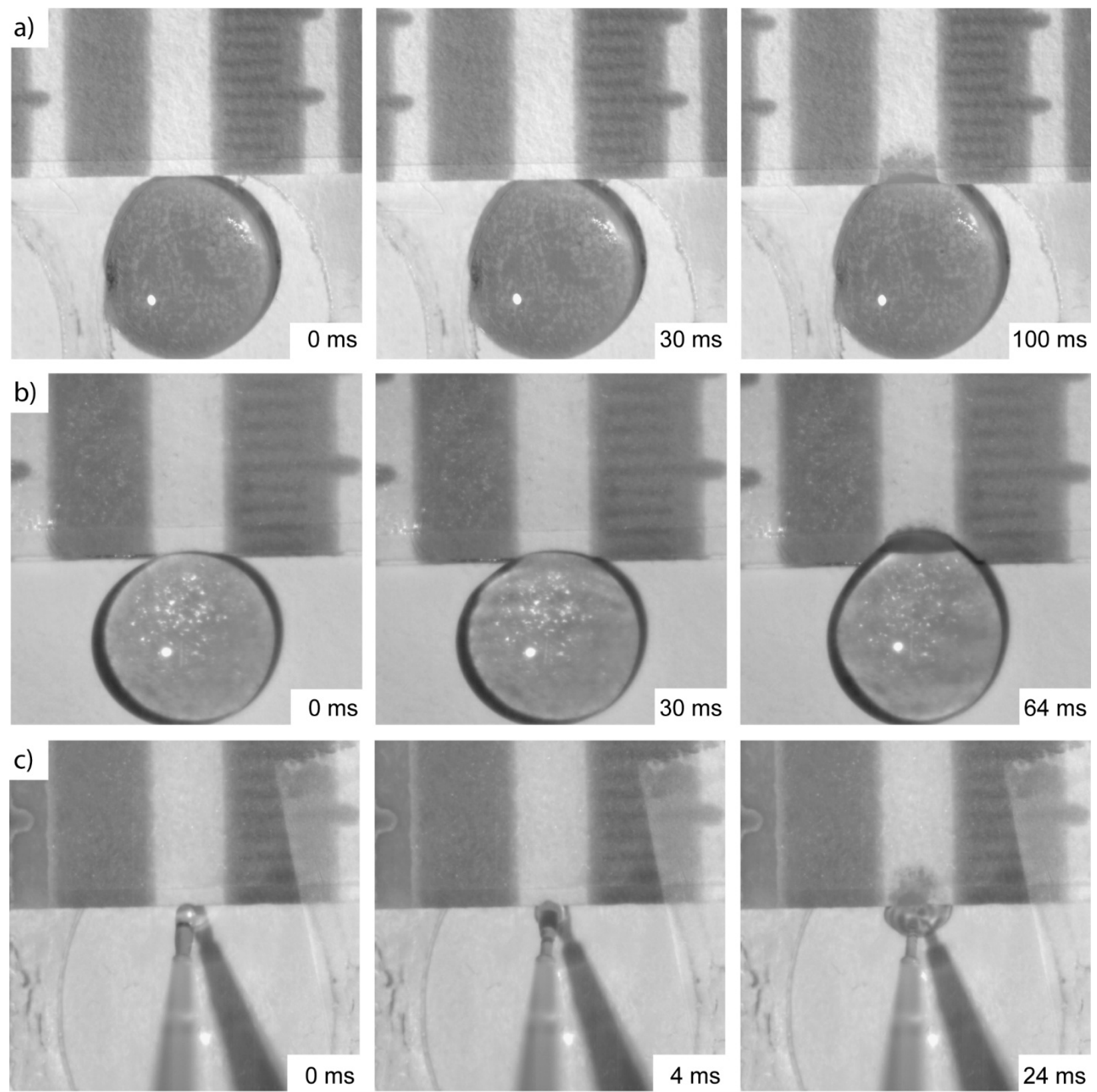

Figure S5. Still images during flow using a) hydrophobic a tape well, b) omniphobic well, and c) pipette sampling at three time points corresponding to the paper contact with the liquid (left column), the liquid encroaching onto the paper device (middle column) and the solution reaching $2 \mathrm{~mm}$ down the channel (right column). 


\section{S4. Evaporation of water from a multilayered $\mu \mathrm{PAD}$}

To estimate the evaporation from a tape-sealed multilayer device during flow, a device was saturated with water, then the mass measured on a balance with time. By subtracting the mass the of the original (dry) device, the mass lost from evaporation of the water (\% mass loss) can be calculated with time, as shown in Figure S6.

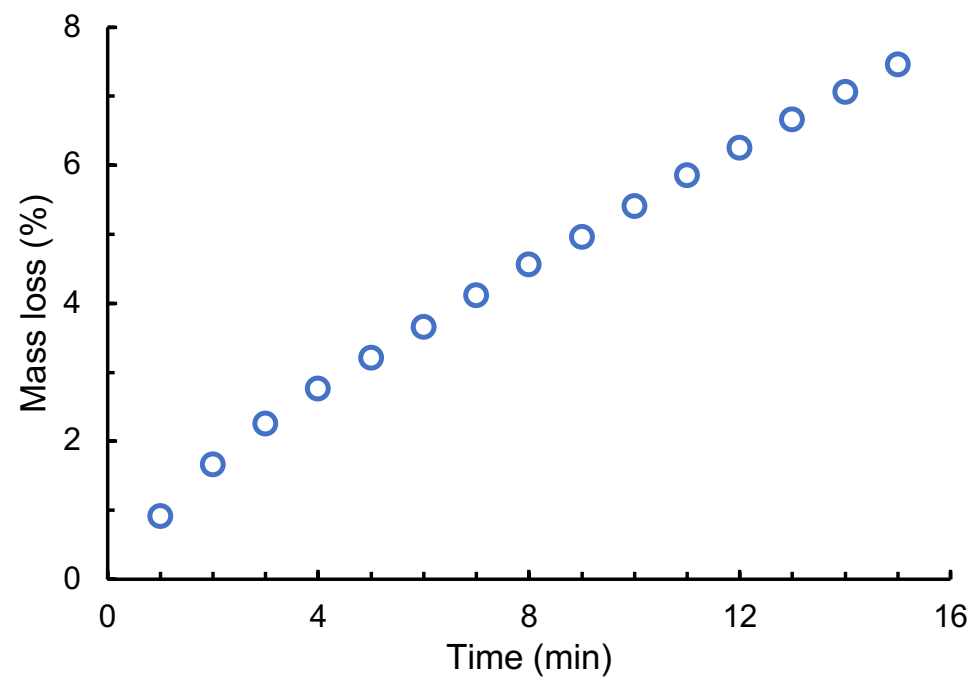

Figure S6. Mass loss-time plot showing the evaporation of water from a multilayered $\mu P A D$, lab humidity $30 \%, 390 \mu \mathrm{m}$ channel height device saturated with $200 \mu \mathrm{L}$ distilled water while being placed on an analytical mass balance.

\section{S5. Model Development}

\section{Previous studies}

As noted in the article, refinement of the Lucas-Washburn equation has continued in order to better capture effects such as paper sub-saturation or physical modifications of a system to better tailor flow characteristics. A recent and thorough review of these various activities ${ }^{1}$ highlights the importance of the ongoing theoretical studies and model development and need for accurate predictive capability, and the authors explicitly note in the review that the mechanisms controlling wicking physics remain unclear. Several of the studies cited in the review are worth discussing because of their potential overlap in describing the physics driving flow in the current multilayer device.

In Cummins et al., ${ }^{2}$ the investigators relaxed the classic Lucas-Washburn assumption that the parallel tubes comprising the pore network in paper were equal in diameter. ${ }^{2}$ By assuming a discrete distribution of ten tube radii ranging from 1 to $19 \mu \mathrm{m}$ a model was developed to predict the time dependent saturation of paper. The rationale for the model was that the liquid front in smaller pores would advance ahead of liquid in larger pores due to the increasingly negative capillary pressures as pore radius decreased. It is important to note that the liquid advancing in the smallest pore is not dragging the liquid in the larger pores but is instead pulling liquid from the larger pores. The investigators found that their system of coupled differential Washburn equations containing experimentally derived parameters yielded accurate predictions of the visible wetted front position as a function of time. And although the wetted front was not saturated, it was found that its position was accurately predicted using the classic Lucas-Washburn equation with the measured average pore radius of $5.08 \mu \mathrm{m}$. Thus, even though the front in the smallest pores could advance 
twice as far as in the largest pores at a given time, the net movement of the liquid was well represented using a single average pore radius.

In an analogous study carried out more than a decade earlier, Bico and Quére ${ }^{3}$ modeled liquid rise in two parallel interconnected tubes with significantly different radii. The hypothesis was that capillary flow was predominantly driven by the Laplace pressure in the large tube. And the liquid front in the small tube advanced ahead of the large tube front because its more negative capillary pressure pumped liquid out of the large tube, significantly reducing viscous dissipation. The model predicted that the distance between the two fronts, normalized by forward (small tube) front location, was approximately equal to the ratio of the tube radii. The model predictions were confirmed by placing a porous cylindrical fiber along the inner surface of a larger cylinder and contacting one end of the assembly with a liquid reservoir. The rise versus time of the two fronts were accurately predicted by their model. With regard to the present work it is significant that the experiments validated the assumption that flow was due solely to the capillary pressure driving force in the large tube.

As discussed in this article, the Lucas-Washburn equation has also been extended to predict imbibition rates in a two-layer device. ${ }^{4}$ By stacking two strips of paper and bonding the edges with a thermal adhesive, a gap between the paper layers was created whose height was approximately $6 \%$ of each paper's thickness. Application of the Lucas-Washburn equation with pore size based on paper properties significantly underpredicted imbibition rate. But, treating the gap as a collection of circular pores and using a cross sectional area-weighted average to obtain an effective pore radius, it was found that the LucasWashburn equation yielded reasonable predictions for penetration depth versus time in the two-layer system.

The work by Camplisson et al. ${ }^{4}$ was not the first to demonstrate the acceleration of flow due to the presence of a gap adjacent to a paper layer. Jahanshahi-Anbuhi et al. ${ }^{5}$ constructed a multi-layer device with a paper layer separated from a flexible PET film by a small gap. They found the presence of the gap increased the flow rate of water through paper by more than a factor of ten. However, this phenomenon is due not only to the adjacent gap but also the deformability of the gap boundary. An earlier study ${ }^{6}$ used parallel plates, one of which was flexible, to demonstrate that capillary rise in the vertical gap was significantly faster and longer lasting than capillary rise between two rigid boundaries. They demonstrated, theoretically and experimentally, that the liquid rising between the two plates exerts a negative pressure that causes the flexible boundary to collapse. Near the middle of the channel the deformable film contacts the rigid boundary (or paper ${ }^{5}$ ). Close to the sides of the channel the boundaries separate and form a wedge, in which the liquid is confined within the infinitesimal gap with a correspondingly small interfacial radius of curvature. Consequently, the driving force for flow is enormous and it greatly exceeds the body force exerted by gravity. Thus, while the flow in these systems is driven by capillary forces in the gap, the mechanics are not governed directly by the initial gap thickness. Interestingly, while the Lucas-Washburn

result shows that penetration distance scales with time as $\ell \propto t^{1 / 2}$, the analysis of wedge flows in deformable boundary devices yields $\ell \propto t^{1 / 3}$.

\section{Models based on published theory}

A number of the cases discussed above suggest how flow behavior in the current multilayer device might be analyzed and explained. These all represent variations on a theme in which the Lucas-Washburn equation is assumed to apply under different scenarios. Since the Lucas-Washburn and derivative equations were developed to describe flow in porous media, it is not obvious that any should apply to a system with a 
distinct homogenous, non-porous flow domain. The reason for the focus on Lucas-Washburn in this study is that the flow is inarguably capillary driven, and the observations follow consistent dependencies. For example, as illustrated in Figure 2, the observed penetration velocity exhibits the expected $u \propto \ell^{-1}$ behavior. Results are also consistent with the well-known $\ell^{2} \propto t$ response (data not shown).

As a baseline case, if we make the erroneous assumption that the liquid in the gap is a passive component of the system and its relatively large size results in negligible viscous dissipation, then flow will be controlled by capillary forces in the paper. In that scenario, as the liquid wicks through the paper, continuity requires it to drag the liquid in the gap along with it. The corresponding predicted velocity to $5.55 \mathrm{~cm}$ in Figure S7 is labeled LW (grey filled diamonds). For the smallest two gap heights the values are not significantly different from observation (purple $\times$ symbols).

The second case represents the other extreme. Building on the results of Bico and Quére, ${ }^{3}$ it was assumed here that flow through the device is driven by capillary forces in the gap. The liquid in the paper layers was ignored in terms of impact on overall flow dynamics. For that situation, the Lucas-Washburn result for capillary-driven flow in a two-dimensional slit is given by

$$
\ell(t)=\sqrt{\frac{2 \gamma h^{2} t \cos \theta}{3 \mu r}}
$$

where $r=h / \cos \theta$, and $\theta$ is the macroscopic contact angle of the liquid with the paper boundary. Based on analyzing a number of still images such as in Figs. 3a and S2 it was determined that $\theta=34 \pm 4^{\circ}$. Application of Equation (S1) at different values of $t$ yielded the filled red circle symbols labeled 'gap only' in Figure S7. This approach grossly overpredicts the flow rate through the device, clearly demonstrating that flow through the paper plays an important role in determining overall flow rate. One aspect of the Bico and Quére hypothesis not captured in this analysis is the assumption that, although flow is driven by the gap Laplace pressure, liquid is being pumped from the gap into the porous layers due to their pressure differentials. When that phenomenon is taken into account, the filled triangle symbols in Figure S7 labeled modified gap are obtained.
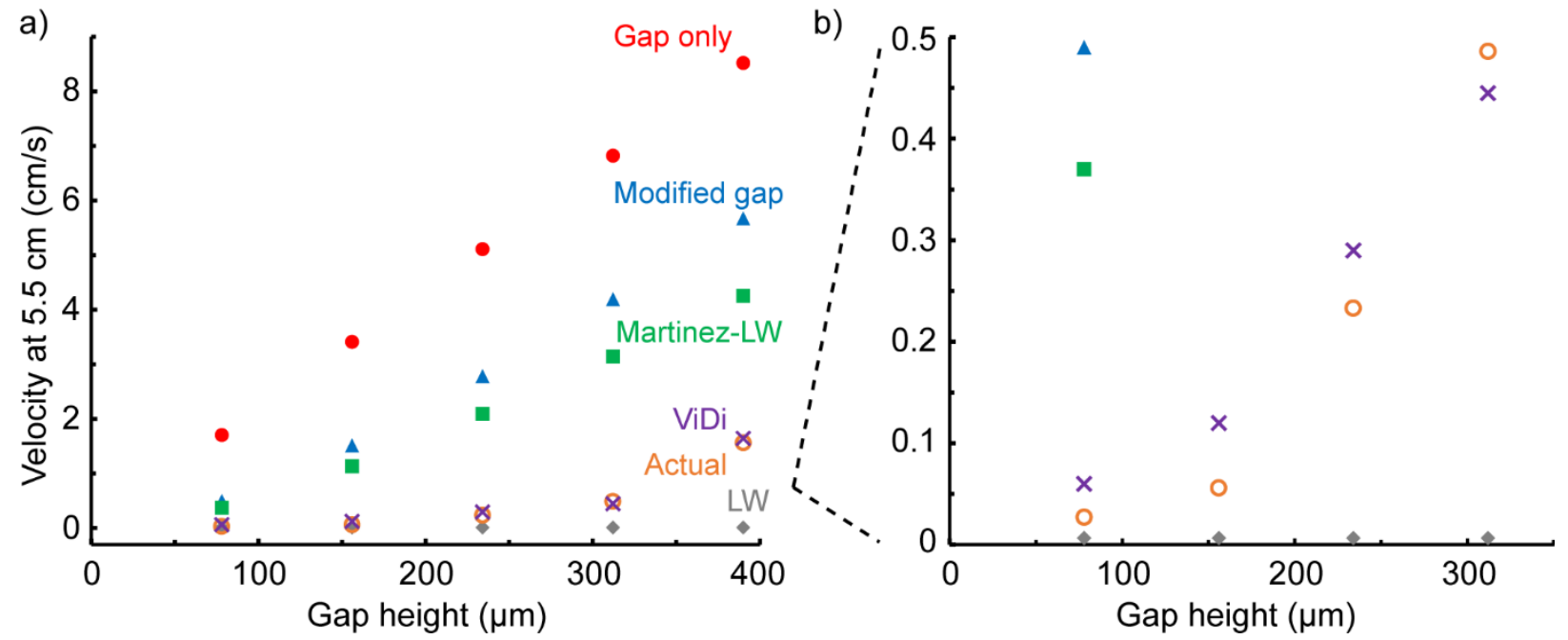

Figure S7. a) A velocity-gap height plot comparing the different models described in this section versus the actual experimentally-measured values with b) a zoom in on the lower velocity data. These data correspond with Figure 5 in the main text. The effective capillary pore size used in each model is shown in Table S1. 
If paper-driven and gap-driven flows represent the two extremes of flow characteristics, then examining an intermediate scenario in which both regions collaboratively contribute to flow is warranted. Specifically, although the focus of Cummins et al. ${ }^{2}$ was modeling the dynamics of saturation in a single paper layer, the study did demonstrate that an appropriate average pore size could be used in the LucasWashburn equation to accurately predict imbibition rates. Similarly, Camplisson et al. ${ }^{4}$ showed that an effective pore size could be used in the same equation to predict imbibition rates for a two-layer paper device containing a thin gap $(\sim 12 \mu \mathrm{m})$. In both studies the appropriate pore size turned out to be a cross sectional area-weighted average of known or assumed pore sizes in each device. Those successful applications of the Lucas-Washburn equation led to predictions of imbibition rate in the current device at the different gap heights. The filled green square symbols in Figure S7 labeled Martinez-LW demonstrate that the area average approach of Camplisson et al. ${ }^{4}$ still significantly overestimates the penetration rate of the liquid through the device.

Table S1. The effective pore size used to obtain the model results plotted in Figure S7. $r^{\prime}$ is the intrinsic average pore size of the paper, $h$ is the gap half-height, $t_{h}$ is the paper thickness, and $\theta$ the contact angle between the liquid in the gap and the paper.

\begin{tabular}{l|c} 
Model & $\boldsymbol{r}$ (effective pore size) \\
\hline Lucas Washburn (LW) & $r^{\prime}$ \\
Gap only & $\frac{h / \cos \theta}{2 h+2 t_{h}}$ \\
Modified gap & $\frac{h^{2}}{2 r^{\prime} t_{h}+2 h^{2}}$ \\
$\begin{array}{l}\text { Martinez-Lucas Washburn } \\
\text { (Martinez-LW) }\end{array}$ & $h / \cos \theta$
\end{tabular}

\section{Current theoretical development - the ViDi model}

The absence of agreement between extensions to the Lucas-Washburn equation and observations led to a re-examination of the physics governing flow dynamics in the multilayer system. Equation (7) in the article is the differential equation that results from incorporating the viscous dissipation-modified pressure driving force into the classic Lucas-Washburn analysis, in this case applied to flow in a horizontal slit rather than in a tube of circular cross section. To obtain Equation (7) we start with the one-dimensional velocity profile for forced flow in a gap or slit with width much greater than its height, which is given by

$$
u_{x}=\frac{G}{2 \mu}\left(y^{2}-h^{2}\right)
$$

where $\mu$ is the liquid viscosity $(\mathrm{Pa} \cdot \mathrm{s}), G$ is the driving force $(\mathrm{Pa} / \mathrm{m})$, and $y$ is the distance measured from the centerline of the gap. Here the device is horizontal, so there is no body force and the flow is purely pressure driven. From Equation (S2) we can obtain an expression for the average velocity $u$ by computing the volumetric flow rate $Q\left(\mathrm{~m}^{3} / \mathrm{s}\right)$ as 


$$
Q=2 w \int_{0}^{h} u_{x} d y=-\frac{2 w G h^{3}}{3 \mu}
$$

where $w$ is the device width, and applying the definition that

$$
u=\frac{Q}{2 h w}=-\frac{G h^{2}}{3 \mu} \text {. }
$$

For negligible viscous dissipation effects, which would be a reasonable assumption within the gap, there would be a correspondingly negligible pressure drop from inlet to liquid front relative to the pressure drop across the curved interface. However, because of the large velocity gradient through the paper thickness it is necessary to take this phenomenon into account. For this we turn to the macroscopic mechanical energy balance. ${ }^{7}$ Drawing a control volume around the paper layer from the device inlet to the liquid front, and assuming (1) no change in potential energy because the device is horizontal, (2) no shaft work, and (3) incompressible flow, the macroscopic mechanical energy balance reduces to

$$
\dot{m} \Delta\left(\frac{1}{2} u^{2}+\frac{p}{\rho}\right)=-\int_{V} \tau: \nabla \mathbf{u} d V
$$

where $\dot{m}$ is the instantaneous mass flow rate $(\mathrm{kg} / \mathrm{s}), \Delta$ represents the difference in value between the inlet and liquid front, $p$ is the pressure $(\mathrm{Pa}), \rho$ is the liquid density $\left(\mathrm{kg} / \mathrm{m}^{3}\right), V$ is the total volume of liquid in the paper $\left(\mathrm{m}^{3}\right), \mathbf{u}$ is the liquid velocity vector in the paper $(\mathrm{m} / \mathrm{s})$, and $\tau$ is the viscous stress tensor (Pa) such that

$$
\tau: \nabla \mathbf{u}=-\frac{1}{2} \mu \sum_{i} \sum_{j}\left(\frac{\partial u_{i}}{\partial x_{j}}+\frac{\partial u_{j}}{\partial x_{i}}\right)^{2}
$$

with the notation that $i, j=1,2,3$ represent the three coordinate directions. As noted in the article, for unidirectional flow with velocity in the $x$ direction that varies in the $y$ direction, Equation (S6) reduces to

$$
\tau: \nabla \mathbf{u}=-\mu\left(\frac{d u_{x}}{d y}\right)^{2}
$$

Since the cross-sectional area of the paper is assumed constant and flow is incompressible, $\Delta\left(u^{2}\right)=0$ in Equation (S5). Noting that $Q=\dot{m} / \rho$, Equations (S5) and (S7) may be written as

$$
\Delta p=P_{v}=\frac{1}{Q} \int_{V} \mu\left(\frac{d u_{x}}{d y}\right)^{2} d y
$$

where $P_{v}$ is the pressure drop in the paper layer from inlet to liquid front due to viscous dissipation effects. This is the same pressure drop experienced by the liquid in the gap, because the assumption of unidirectional flow (in the $x$-direction) reduces the $y$-component of the Navier-Stokes equations to the trivial expression $\partial p / \partial y=0$ in the device (neglecting body force). That is, pressure is a function of only $x$ in the paper and gap. Then, the driving force for flow in Equation (S2) is $G=-\Delta P / \ell$, where the net pressure difference driving force is

$$
\Delta P=P_{c}-P_{v}=\frac{\gamma}{r} \cos \theta-\frac{1}{Q} \int_{V} \mu\left(\frac{d u_{x}}{d y}\right)^{2} d V
$$

which is Equation (5) in the article. In Equation (S9), $\gamma$ is the interfacial tension ( $\mathrm{N} / \mathrm{m}), r=h / \cos \theta$ is the radius of curvature of the liquid front, $\mu$ is the liquid viscosity, and $V$ is the volume of liquid in the paper. 
Once Equation (6) is substituted into Equation (S4) we obtain Equation (7) which, as noted in the article, is a form of the d'Alembert equation, named after the French mathematician, based on his extensive studies of its singularity behavior. That equation is of the general form

$$
x\left(\frac{d y}{d x}\right)^{2}+a y \frac{d y}{d x}=c
$$

or

$$
x\left(y^{\prime}\right)^{2}+a y y^{\prime}=c
$$

where $y^{\prime}=d y / d x$. To solve this equation, it must first be manipulated to obtain a tractable form. We first subtract $x\left(y^{\prime}\right)^{2}$ from both sides and divide by $a y^{\prime}$. This leads to

$$
y=-\frac{x y^{\prime}}{a}+\frac{c}{a y^{\prime}} \text {. }
$$

We then define $p=y^{\prime}$ so that Equation (S11) may be written as

$$
y=x f(p)+g(p)
$$

where $f(p)=-p / a$ and $g(p)=c / a p$. We then differentiate Equation (S12) with respect to $x$ to get

$$
p=f(p)+x f^{\prime}(p) \frac{d p}{d x}+g^{\prime}(p) \frac{d p}{d x} .
$$

After a little bit of algebra and multiplying the equation by $(d x / d p)$ we get

$$
\frac{d x}{d p}+\frac{x}{p(a+1)}=-\frac{c}{p^{3}(a+1)}
$$

This equation is linear in $x$ as a function of $p\left(=y^{\prime}=d y / d x\right)$ and can be solved using standard techniques. First an integration factor is defined as

$$
\xi=\exp \left(\int \frac{d p}{p(a+1)}\right)=\sqrt[a+1]{p(a+1)} .
$$

Multiply both sides of Equation (S13) by $\xi$ to get

$$
\sqrt[a+1]{p(a+1)} \frac{d x}{d p}+\frac{x \sqrt[a+1]{p(a+1)}}{p(a+1)}=-\frac{c \sqrt[a+1]{p(a+1)}}{p^{3}(a+1)} .
$$

Noting that

$$
\frac{\sqrt[a+1]{p(a+1)}}{p(a+1)}=\frac{d}{d p}(\sqrt[a+1]{p(a+1)})
$$

in Equation (S14), a substitution and use of the reverse product rule yields

$$
\frac{d}{d p}(x \sqrt[a+1]{p(a+1)})=-\frac{c \sqrt[a+1]{p(a+1)}}{p^{3}(a+1)} .
$$


Both sides of Equation (S15) may now be integrated with respect to $p$, and the indefinite integral is

$$
x \sqrt[a+1]{p(a+1)}=\frac{c \sqrt[a+1]{p(a+1)}}{p^{2}(2 a+1)}+\beta
$$

where $\beta$ is an arbitrary constant. Dividing both sides of Equation (S16) by $\xi=\sqrt[a+1]{p(a+1)}$ we obtain

$$
x=\frac{c}{p^{2}(2 a+1)}+\frac{\beta}{\sqrt[a+1]{p(a+1)}} .
$$

Equation (S17) can be used directly, since when it is converted to the variables associated with Equation (7) we immediately obtain

$$
t=\frac{c}{(2 a+1) u^{2}}+\frac{\beta}{\sqrt[a+1]{(a+1) u}} .
$$

To determine the constant $\beta$ we apply the initial condition that $u=u_{0}$ at $t=0$. It is found that

$$
\beta=-\frac{c\left[(a+1) u_{0}\right]^{1 /(a+1)}}{(2 a+1) u_{0}^{2}}
$$

and when substituted into Equation (S18) and simplified, the result is

$$
t=\frac{c}{2 a+1}\left[\frac{1}{u^{2}}-\frac{1}{u_{0}^{2}}\left(\frac{u_{0}}{u}\right)^{1 /(a+1)}\right]
$$

which is Equation (10), and it provides a way to compute velocity as a function of time.

If instead the goal is to calculate penetration distance $\ell$ as a function of time we note that, since $p=y^{\prime}$, Equation (S10) may also be written as $x p^{2}+a y p-c=0$, which has a single physical root given by

$$
p=\frac{1}{2 x}\left(-a y+\sqrt{a^{2} y^{2}+4 x c}\right) .
$$

When Equation (S21) is substituted into Equation (S17) the result is

$$
x=\frac{4 c x^{2}}{(2 a+1)\left[\sqrt{4 c x+a^{2} y^{2}}-a y\right]^{2}}+\beta\left[\frac{x}{(a+1)\left(\sqrt{4 c x+a^{2} y^{2}}-a y\right)}\right]^{1 /(a+1)}
$$

where $\beta$ will have a different value than in Equations (S18) and (S19), and would be specified by applying a similar constraint as before, in this case the constraint that $\ell=0$ at $t=0$, which in the context of Equation (S22) is $y=0$ at $x=0$. This step has not yet been carried out. 


\section{REFERENCES}

1. Liu, Z.; He, X.; Han, J.; Zhang, X.; Li, F.; Li, A.; Qu, Z.; Xu, F., Liquid wicking behavior in paper-like materials: mathematical models and their emerging biomedical applications. Microfluidics and Nanofluidics 2018, 22 (11), 132.

2. Cummins, B. M.; Chinthapatla, R.; Ligler, F. S.; Walker, G. M., Time-Dependent Model for Fluid Flow in Porous Materials with Multiple Pore Sizes. Anal. Chem. 2017, 89 (8), 4377-4381.

3. Bico, J.; Quéré, D., Precursors of impregnation. EPL (Europhysics Letters) 2003, 61 (3), 348.

4. Camplisson, C. K.; Schilling, K. M.; Pedrotti, W. L.; Stone, H. A.; Martinez, A. W., Two-ply channels for faster wicking in paper-based microfluidic devices. Lab on a Chip 2015, 15 (23), 4461-4466.

5. Jahanshahi-Anbuhi, S.; Chavan, P.; Sicard, C.; Leung, V.; Hossain, S. M. Z.; Pelton, R.; Brennan, J. D.; Filipe, C. D. M., Creating fast flow channels in paper fluidic devices to control timing of sequential reactions. Lab on a Chip 2012, 12 (23), 5079-5085.

6. Cambau, T.; Bico, J.; Reyssat, E., Capillary rise between flexible walls. EPL (Europhysics Letters) 2011, 96 (2), 24001.

7. Bird, R.B.; Stewart, W.E.; Lightfoot, E.N.; Klingenberg, D.J., Introductory Transport Phenomena, John Wiley \& Sons: 2015; p. 193. 